\title{
Plumbagin induces apoptosis in human osteosarcoma through ROS generation, endoplasmic reticulum stress and mitochondrial apoptosis pathway
}

\author{
CHIA-CHIA CHAO ${ }^{1}$, SHENG-MOU HOU ${ }^{2}$, CHIEH CHEN HUANG ${ }^{3}$, \\ CHUN-HAN HOU ${ }^{4}$, PO-CHUN CHEN ${ }^{5,6}$ and JU-FANG LIU ${ }^{7}$
}

\begin{abstract}
${ }^{1}$ Department of Respiratory Therapy, Fu-Jen Catholic University, New Taipei 24257; ${ }^{2}$ Department of Orthopedic Surgery, Shin-Kong Wu Ho-Su Memorial Hospital, Taipei 11101; ${ }^{3}$ Department of Life Sciences, National Chung Hsing University, Taichung 40227; ${ }^{4}$ Department of Orthopedic Surgery, National Taiwan University Hospital, Taipei 10002; ${ }^{5}$ Graduate Institute of Basic Medical Science, China Medical University, Taichung 40402; ${ }^{6}$ Department of Medical Research, Chung Shan Medical University Hospital, Chung Shan Medical University, Taichung 40201; ${ }^{7}$ Central Laboratory, Shin-Kong Wu Ho-Su Memorial Hospital, Taipei 11101, Taiwan R.O.C.
\end{abstract}

Received August 11, 2016; Accepted May 9, 2017

DOI: $10.3892 / \mathrm{mmr} .2017 .7222$

\begin{abstract}
Osteosarcoma is the most common primary bone tumor that occurs in children and adolescents. Osteosarcoma has a poor prognosis and is often unresponsive to chemotherapy. Therefore, it remains a challenge to identify a novel strategy to effectively treat osteosarcoma. The present study demonstrated a novel opportunity in osteosarcoma treatment using the natural compound plumbagin. Plumbagin reduced cell viability in osteosarcoma cells but not normal bone cells, as determined by MTT assay and colony formation assay. Plumbagin induced cell apoptosis by mitochondrial dysfunction, which in turn promoted $\mathrm{Ca}^{2+}$ release and endoplasmic reticulum (ER)-stress, as determined by DAPI staining assay, DNA fragmentation assay, flow cytometry and western blotting analysis. In addition, plumbagin improved reactive oxygen species (ROS) generation, as determined by flow cytometry. Finally, these apoptotic cascades activated caspase-3 and caspase-9 to elicit apoptosis response. Our results demonstrated the anticancer effect of plumbagin by inducing cell apoptosis in osteosarcoma cells. In conclusion, plumbagin activated the apoptosis signaling pathway through eliciting ROS, ER stress, mitochondria dysfunction, and finally causing caspase activation. These results indicated that plumbagin may serve as potential antitumor drug by its multifunctional effects in osteosarcoma.
\end{abstract}

Correspondence to: Dr Ju-Fang Liu, Central Laboratory, Shin-Kong Wu Ho-Su Memorial Hospital, 95 Wenchang Road, Shilin, Taipei 11101, Taiwan R.O.C.

E-mail: T010615@ms.skh.org.tw

Key words: osteosarcoma, plumbagin, endoplasmic reticulum stress, apoptosis

\section{Introduction}

Osteosarcoma is the most common primary bone tumor occurring in adolescents. To date, conventional therapies containing chemotherapy and radiation treatment have had a poor response. Moreover, pulmonary metastasis is responsible for the high mortality in osteosarcoma patients (1). Therefore, it is important to develop an alternative strategy in osteosarcoma treatment. Previously, natural compounds have been implicated in tumor therapy by its antitumor effects to suppress tumor proliferation or induce apoptosis of tumor cells (2).

Plumbago zeylanica L. (also known as 'Chitrak') is a traditionally used medicinal plant that has numerous biological functions such as anticarcinogenic, antiatherosclerotic and antimicrobial effects (3-5). Plumbagin (5-hydroxy-2-methyl-1,4-naphthoquinone), a quinonoid constituent isolated from the root of Plumbago zeylanica (Fig. 1A) shows antitumor effects in various tumors, for example breast cancer (6), cervical cancer (4), prostate cancer (7) and lung cancer (8). However, little is understood regarding the mechanism of plumbagin-induced antitumor effects in osteosarcoma.

Dysregulation of cell apoptosis, also named 'programmed cell death', is an important characteristic in tumor progression. Numerous natural compounds bring about their antitumor effects by inducing apoptosis in cancer cells (9). There are two major mechanisms involved in apoptosis regulation. One is the extrinsic pathway, which is elicited by the Fas death receptor, a member of the tumor necrosis factor receptor superfamily (10). The other is the intrinsic pathway, in which the mitochondria serve a central role by releasing cytochrome c (11). Finally, these signals activate a series of proteases named caspases. For example, the intrinsic and extrinsic apoptotic pathways activate caspase-3 and lead to DNA condensation and fragmentation (12). Previously, ROS have also been implicated in apoptosis induction through the activation of the mitochondrial-dependent cell death 
pathway (13). Discovery of mechanisms involved in apoptosis provides an opportunity for novel targeted therapies that promote death in cancer cells.

In the present study, the authors reported the antitumor effect of plumbagin in osteosarcoma cells. The results indicated that plumbagin induces cell apoptosis by inducing the ROS-related mitochondria-dependent cell death pathway of human osteosarcoma cells.

\section{Materials and methods}

Materials. Horseradish peroxidase-conjugated anti-mouse and anti-rabbit IgG, and rabbit polyclonal antibodies specific for $\beta$-actin (Sigma-Aldrich; Merck KGaA, Darmstadt, Germany; cat. no. SI-A5441; 1:10,000), voltage-dependent anion channel (GeneTex International Corporation, Hsinchu, Taiwan; cat. no. GTX-104745; 1:1,000), cytochrome c (GeneTex International Corporation; cat. no. GTX-108580; 1:1,000), B cell lymphoma (Bcl)-2 (GeneTex International Corporation; cat. no. GTX-100064; 1:1,000), Bcl-xL (GeneTex International Corporation; cat. no. GTX-105661; 1:1,000), Bcl-2-associated X (Bax; GeneTex International Corporation; cat. no. GTX-109683; 1:1,000), Bcl-2 homologous antagonist/killer (Bak; GeneTex International Corporation; cat. no. GTX-100063; 1:1,000), 78 kDa glucose-regulated protein (GRP78) (GeneTex International Corporation; cat. no. GTX113340; 1:1,000), GRP94 (GeneTex International Corporation; cat. no. GTX103203; 1:1,000), caspase-3 (Santa Cruz Biotechnology, Inc., Dallas, TX, USA; cat. no. SC-7272; 1:1,000), caspase-9 (Santa Cruz Biotechnology, Inc.; cat. no. SC-56073; 1:1,000) and poly (ADP-ribose) polymerase (PARP; GeneTex International Corporation; cat. no. GTX100573, 1:1,000) were used in the present study. Plumbagin from Plumbago indica and all other chemicals were obtained from Sigma-Aldrich; Merck KGaA.

Cell culture. The human osteosarcoma cell lines (MG-63, U2OS, HOS) and osteoblast cell line (hFOB 1.19) were purchased from American Type Culture Collection (Manassas, VA, USA). MG-63, U2OS, HOS and hFOB 1.19 cells were maintained in an $\alpha$-modified Eagle's medium (MEM), McCoy's 5A, $\alpha$-MEM and DMEM/Ham's F-12 medium, respectively (all Invitrogen; Thermo Fisher Scientific, Inc., Waltham, MA, USA). All cells were incubated at $37^{\circ} \mathrm{C}$ in an atmosphere of $5 \% \mathrm{CO}_{2}$ supplemented with $20 \mathrm{mM}$ hydroxyethyl piperazineethanesulfonic acid (HEPES; Sigma-Aldrich; Merck KGaA), $10 \%$ heat-inactivated fetal calf serum (FBS), $2 \mathrm{mM}$ of glutamine, $100 \mathrm{U} / \mathrm{ml}$ penicillin and $100 \mu \mathrm{g} / \mathrm{ml}$ of streptomycin (all Invitrogen; Thermo Fisher Scientific, Inc.). The media were changed every $48 \mathrm{~h}$.

Cell viability assay. Cell viability was determined by MTT assay. Following treatment with indicated condition of plumbagin for 1 or 2 days, cultures were washed with PBS. All cells were incubated with MTT $(0.5 \mathrm{mg} / \mathrm{ml})$ for $2 \mathrm{~h}$ at $37^{\circ} \mathrm{C}$. Following incubation, dimethylsulfoxide was added to dissolve formazan crystals and shaken at room temperature for $10 \mathrm{~min}$. Finally, the cell viability was assessed by the absorbance of $570 \mathrm{~nm}$ using a microplate reader (Bio-Tek Instruments, Winooski, VT, USA).
Colony formation assay. The cells were seeded in six-well plates $\left(1 \times 10^{3}\right.$ cells/well) and incubated with indicated condition of plumbagin $(1,2.5,5$ and $10 \mu \mathrm{M})$ for 10 days. Culture media were changed every 2 days. At 10 days, colonies were washed with PBS, fixed for 15 min with $4 \%$ paraformaldehyde and stained with $0.1 \%$ crystal violet for $5 \mathrm{~min}$. The colony forming cells were photographed. Following undergoing three washes with $\mathrm{ddH}_{2} \mathrm{O}$, acetic acid was added to a final concentration of $33 \%(\mathrm{v} / \mathrm{v})$, which was achieved followed by measuring the absorbance at $550 \mathrm{~nm}$. The colony formation assay was repeated three times with duplicate wells.

DAPI staining. The cells were treated with indicated condition of plumbagin for $48 \mathrm{~h}$, the cells were washed with PBS, fixed in a $3.7 \%$ formaldehyde solution for $10 \mathrm{~min}$, and stained with DAPI $(0.5 \mu \mathrm{g} / \mathrm{ml})$ for $5 \mathrm{~min}$. The cells were photographed using fluorescence microscopy.

DNA ladder assay. The cells were treated with previously indicated conditions of plumbagin for $48 \mathrm{~h}$. Total DNA were extracted and used to agarose electrophoresis (1.5\% agarose gel). The agarose gel was stained with ethidium bromide to assess the DNA fragmentation. Ultraviolet spectroscopy at $302 \mathrm{~nm}$ was used to report results.

Western blot analysis. Total cell lysates were extracted as described previously (14). Proteins were resolved on a $10-15 \%$ SDS-PAGE and transferred to polyvinylidene membranes. The blots were blocked with $4 \%$ bovine serum albumin (cat. no. A9647; Sigma-Aldrich; Merck KGaA), for $1 \mathrm{~h}$ at room temperature and then probed with first antibodies $(1: 1,000)$ for $1 \mathrm{~h}$ at room temperature. Following three washes, the blots were subsequently incubated with peroxidase-conjugated secondary antibodies $(1: 1,000)$ for $1 \mathrm{~h}$ at room temperature. The blots were visualized by enhanced chemiluminescence using Kodak X-OMAT LS film (Kodak, Rochester, NY, USA). The bands were analyzed using ImageJ software (version 1.51k; https://imagej.nih.gov/ij/). Each experiment was repeated at least three times.

Annexin V/propidium iodide (PI) staining. Apoptosis was assessed using Annexin V, a protein that binds to phosphatidylserine residues which are exposed on the cell surface of apoptotic cells, as previously described (15). Cells ( $2 \times 10^{6}$ cells) were treated with indicated conditions of plumbagin. Following treatment, cells were washed twice with PBS, and resuspended in staining buffer containing $1 \mu \mathrm{g} / \mathrm{ml}$ PI and $0.025 \mu \mathrm{g} / \mathrm{ml}$ annexin V-fluorescein isothiocyanate (FITC). Double-labeling was performed at room temperature for $10 \mathrm{~min}$ in the dark prior to flow cytometric analysis. Cells were immediately analyzed using FACScan and the CellQuest pro software (version 5.1; BD Biosciences, Franklin Lakes, NJ, USA).

Cell cycle analysis by PI staining. Quantitative assessment of apoptotic cells was also assessed by examining the cell cycle. Cells were collected by centrifugation $(10 \mathrm{~min}$ at $950 \mathrm{x} \mathrm{g}$ and $4^{\circ} \mathrm{C}$ ) and adjusted to $2 \times 10^{6}$ cells $/ \mathrm{ml}$. Pre-chilled ethanol was added to $0.5 \mathrm{ml}$ cell suspension and the mixture was incubated at $4^{\circ} \mathrm{C}$ for $30 \mathrm{~min}$. Ethanol was then removed by centrifugation $\left(15 \mathrm{~min}\right.$ at $1,425 \mathrm{x} \mathrm{g}$ and $\left.4^{\circ} \mathrm{C}\right)$, and cellular DNA was 

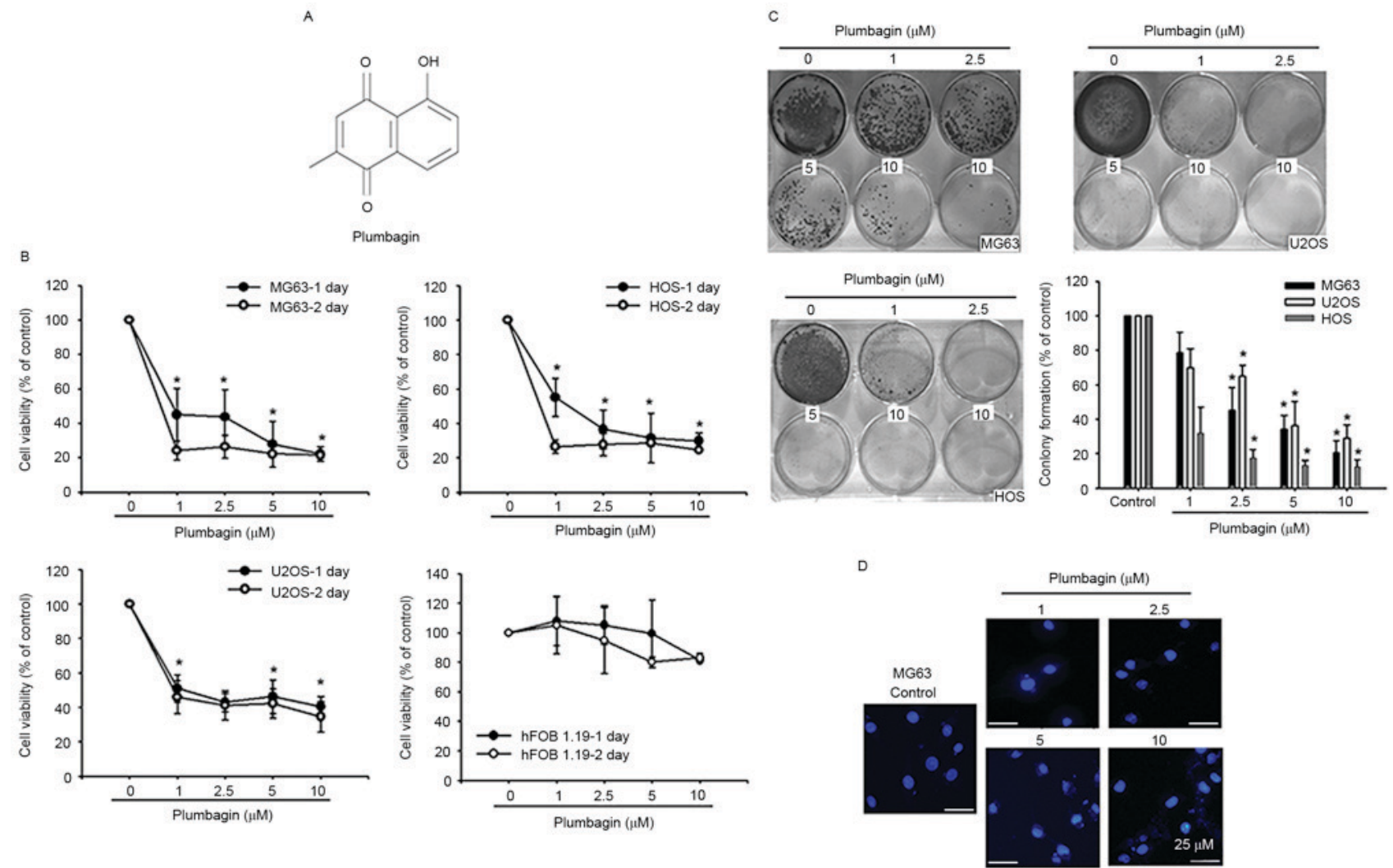

Figure 1. Plumbagin decreases cell viability in osteosarcoma cells. (A) The structure of plumbagin. (B) The MG63, U2OS and HOS osteosarcoma cells and osteoblast cells hFOB 1.19 were treated with indicated concentrations of plumbagin for 1 and 2 day, and cell viability was assessed by MTT assay. (C) The cells were treated incubated with plumbagin for 10 days. The colony forming cells were stained with crystal violet and photographed. The quantitative data are presented in the right-lower panel. (D) MG63 cells were incubated with indicated conditions of plumbagin for 24 h, and the nucleus morphology were assessed by DAPI staining. Magnification, $\mathrm{x} 40$. Results are expressed as the mean \pm standard error of the mean. ${ }^{*} \mathrm{P}<0.05$ vs. controls.

stained with $100 \mu \mathrm{g} / \mathrm{ml}$ PI (in PBS containing $0.1 \%$ Triton-X 100 , and $1 \mathrm{mM}$ EDTA) in the presence of an equal volume of DNase-free RNase A $(200 \mu \mathrm{g} / \mathrm{ml}$; Sigma-Aldrich; Merck $\mathrm{KGaA}$ ). Following staining, cells were analyzed immediately with FACScan and CellQuest programs (version 5.1; BD Biosciences). The extent of apoptosis was determined by measuring the DNA content of cells below sub G1 peak (16).

Terminal deoxynucleotidyl-transferase-mediated dUTP nick end labeling (TUNEL) assay. Quantitative assessment of apoptotic cells was also assessed by TUNEL assay, which examines DNA-strand breaks during apoptosis by using the BD APO-DIRECT ${ }^{\mathrm{TM}}$ kit (BD Biosciences; cat. no. 556381). Briefly, cells $\left(2 \times 10^{6}\right.$ cells) were incubated with plumbagin for the indicated times. The cells were trypsinized and collected by centrifugation $\left(10 \mathrm{~min}\right.$ at $950 \mathrm{x} \mathrm{g}$ and $\left.4^{\circ} \mathrm{C}\right)$. The cells were fixed with $1 \%$ paraformaldehyde for $30 \mathrm{~min}$ on ice. Once the fixative was removed, cells were washed twice with PBS, ethanol was added to the $0.5 \mathrm{ml}$ cell suspension and the mixture was incubated at $-20^{\circ} \mathrm{C}$ for $2 \mathrm{~h}$. Ethanol was then removed by centrifugation $(15 \mathrm{~min}$ at $1,425 \mathrm{x} \mathrm{g}$ and $4^{\circ} \mathrm{C}$ ) and cellular DNA was stained with TUNEL solution ( $3 \mathrm{ng} / \mu 1 \mathrm{TdT}$ enzyme and $0.04 \mathrm{nMol}$ FITC dUTP) for $1 \mathrm{~h}$ at $37^{\circ} \mathrm{C}$. Following incubation, $1 \mathrm{ml}$ Rinse buffer was added and centrifuged again for $15 \mathrm{~min}$ at $1,425 \mathrm{x} \mathrm{g}$ and $4^{\circ} \mathrm{C}$. The fluorescein labels incorporated in DNA strand breaks were detected and quantified immediately with FACScan and
CellQuest programs (version 5.1; BD Biosciences) with a minimum of 10,000 counts/sample.

Determination of mitochondrial membrane potential. The mitochondrial membrane potential $(\Delta \Psi \mathrm{m})$ was assessed using the fluorometric probe Tetraethylbenzimidazolylcarbocyanine iodide (JC-1; EMD Millipore, Billerica, MA, USA), a positively charged mitochondria-specific fluorophore that indicates depolarization by a fluorescence emission shift from green $(525 \mathrm{~nm})$ to red $(610 \mathrm{~nm})(17)$. Briefly, cells $\left(2 \times 10^{6}\right.$ cells $)$ were plated in six-well culture dishes, grown to confluence, and treated with vehicle or plumbagin. Following incubation, cells were stained with $\mathrm{JC}-1(5 \mu \mathrm{g} / \mathrm{ml})$ for $15 \mathrm{~min}$ at $37^{\circ} \mathrm{C}$ and then analyzed by FACScan using an argon laser $(488 \mathrm{~nm})$. Mitochondrial depolarization, which is specifically indicated by a decrease in the red/green fluorescence intensity ratio, was analyzed by CellQuest program (version 5.1; BD Biosciences).

Measurements of reactive oxygen species. Levels of intracellular $\mathrm{H}_{2} \mathrm{O}_{2}$ were assessed spectrofluorimetrically by oxidation of specific probes: $\mathrm{H}_{2}$ DCFDA (Molecular Probes; Thermo Fisher Scientific, Inc.). Cells were plated at a density of $5 \times 10^{5}$, allowed to attach overnight, and exposed to plumbagin for specified time intervals. The cells were stained with $\mathrm{H}_{2} \mathrm{DCFDA}$ $(10 \mu \mathrm{M})$ for $10 \mathrm{~min}$ at $37^{\circ} \mathrm{C}$ and the fluorescence intensity in cells was determined using the flow cytometry. The levels of ROS generation was stained with CellROX ${ }^{\circledR}$ Deep Red reagent 

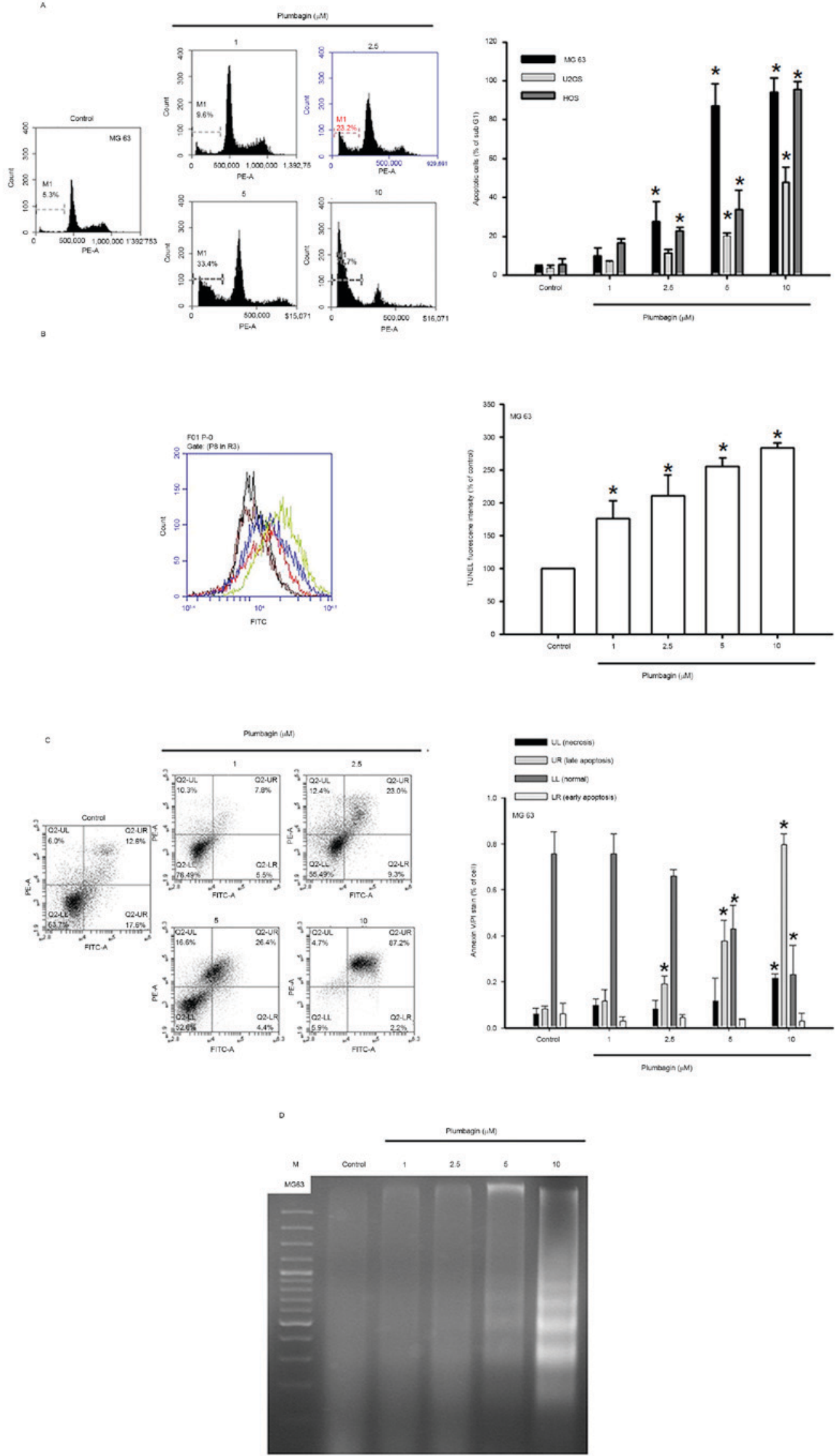

Figure 2. Plumbagin induces cell apoptosis in osteosarcoma cells. (A) The MG63, U2OS and HOS osteosarcoma cells were incubated with indicated conditions of plumbagin for $24 \mathrm{~h}$, the cells were stained by PI and the apoptotic cells were assessed by flow cytometric analysis. (B) MG63 cells were incubated with indicated conditions of plumbagin for $24 \mathrm{~h}$, the cells were stained by TUNEL and percentage of apoptotic cells were analyzed by flow cytometric analysis. (C) MG63 cells were treated as described in (B). The cells were stained by Annexin V/PI and percentage of apoptotic cells were analyzed by flow cytometric analysis. (D) MG63 cells were treated as described in (B), DNA was extracted from total cell lysates and DNA fragmentation was assessed by DNA gel electrophoresis. Results are expressed as the mean \pm standard error of the mean. ${ }^{*} \mathrm{P}<0.05$ vs. controls. PI, propidium iodide; FITC, fluorescein isothiocyanate. 

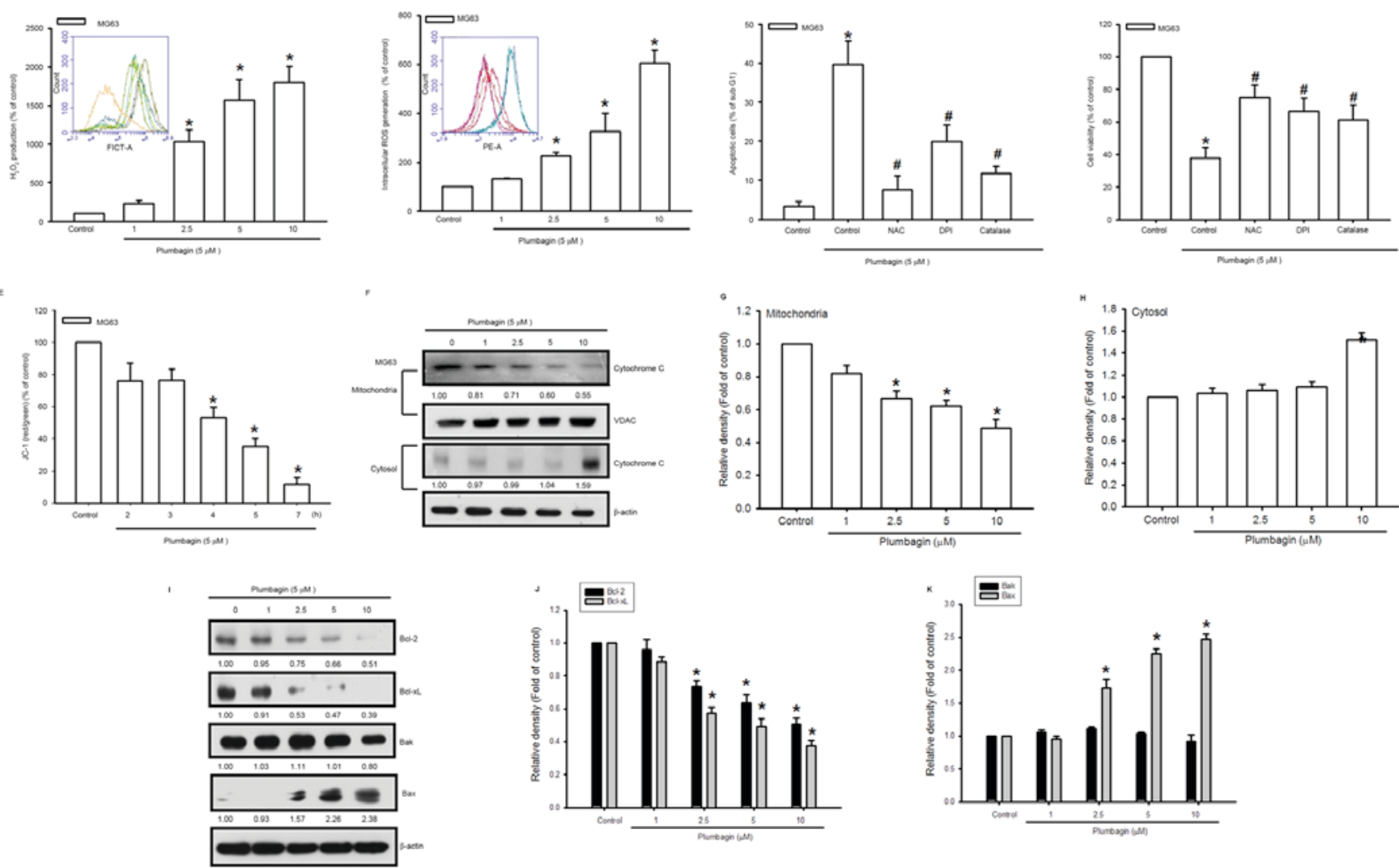

Figure 3. Plumbagin induces ROS-triggered mitochondrial dysfunction in osteosarcoma. (A and B) MG63 cells were treated with indicated concentrations of plumbagin, the $\mathrm{H}_{2} \mathrm{O}_{2}$ production and ROS generation were assessed by H2DCFDA staining kit and CellROX ${ }^{\circledR}$ Deep Red staining, and the stained cells were performed with flow cytometric analysis. (C and D) MG63 cells were pretreated with NAC, DPI and catalase for 30 min, then the cells were incubated with plumbagin for $24 \mathrm{~h}$. The percentage of apoptotic cells and cell viability were assessed by PI staining and MTT assay. (E) MG63 cells were incubated with indicated times of plumbagin, the cells were stained by JC-1 dye and mitochondrial membrane potential was assessed by using flow cytometry. (F) MG63 cells were treated with indicated concentrations of plumbagin for $24 \mathrm{~h}$, the cytosolic and mitochondria fractions were prepared from total cell lysates and cytochrome $\mathrm{c}$ expression levels were assessed by western blot analysis. Actin and VDAC were used as internal control. Quantitation of cytochrome c protein levels in the (G) mitochondria and (H) cytosolic fractions. (I) MG63 cells were treated with the indicated concentrations of plumbagin for $24 \mathrm{~h}$, the total proteins were extracted from the cells and Bcl-2, Bcl-xL, Bak and Bax expression levels were assessed by western blotting. Quantitation of (J) Bcl-2 and $\mathrm{Bcl}-\mathrm{xL}$, and (K) Bak and Bax protein levels in MG63 cells treated with 1, 2.5, 5 and $10 \mu \mathrm{M}$ plumbagin for $24 \mathrm{~h}$. Results are expressed as the mean \pm standard error of the mean. ${ }^{*} \mathrm{P}<0.05$ vs. controls; ${ }^{*} \mathrm{P}<0.05$ vs. plumbagin-treated control. ROS, reactive oxygen species; NAC, N-acetylcysteine; DPI, diphenylene iodonium; JC-1, tetraethylbenzimidazolylcarbocyanine iodide; Bcl, B cell lymphoma; Bak, Bcl-2 homologous antagonist/killer; Bax, Bcl-2-associated X; VDAC, voltage-dependent anion channel.

(Thermo Fisher Scientific, Inc.), and ROS generation was assessed by using the flow cytometry.

Detection of $\mathrm{Ca}^{2+}$ Concentrations. Cells $\left(2 \times 10^{5}\right)$ seeded in 12 -well plates were incubated with plumbagin for indicated times to detect changes in $\mathrm{Ca}^{2+}$ levels. Cells were harvested and washed twice, and re-suspension in Fluo 3/AM $(3 \mu \mathrm{g} / \mathrm{ml}$; Sigma-Aldrich; Merck $\mathrm{KGaA}$ ) at $37^{\circ} \mathrm{C}$ for $30 \mathrm{~min}$ and analyzed by flow cytometry. For $\mathrm{Ca}^{2+}$ expression, the cells were stained with Calcium Assay kit (Cayman Chemical Company, Ann Arbor, MI, USA) and $\mathrm{Ca}^{2+}$ expression was assessed by using flow cytometry.

Caspase activity assay. The assay is based on the ability of the active enzyme to cleave the chromophore from the enzyme substrate Ac-LEHD-pNA (for caspase-9; cat. no. APT173; EMD Millipore) and Ac-DEVD-pNA (for caspase-3; cat. no. APT131; EMD Millipore). The cell lysates were prepared and incubated with the specific anti-caspase- 9 and caspase- 3 substrate solution (final substrate concentration of $0.3 \mathrm{mg} / \mathrm{ml}$ ) for $2 \mathrm{~h}$ at $37^{\circ} \mathrm{C}$. The release of p-nitroaniline was monitored at $405 \mathrm{~nm}$. Results are represented as the percentage change of the activity compared to the untreated control.

Migration and invasion assay. The upper chambers were coated with BD Matrigel Basement Membrane Matrix (50 $\mu \mathrm{g} / \mathrm{ml}$; BD Biosciences). Cells $\left(1 \times 10^{4}\right)$ in serum-free medium were added to the upper chamber of a Transwell insert, and vehicle control or plumbagin $(1,2.5,5$ and $10 \mu \mathrm{M})$ in serum-free medium was applied to the lower chamber, followed by incubation. At $18 \mathrm{~h}$, cells were fixed with $3.7 \%$ formaldehyde and stained with $0.05 \%$ crystal violet. Stained cells that migrated to the lower chamber were counted.

Statistical analysis. Data are presented as the mean \pm standard error of the mean. Statistical comparisons between two groups were performed using the Student's $t$ test. $\mathrm{P}<0.05$ was considered to indicate a statistically significant difference. 

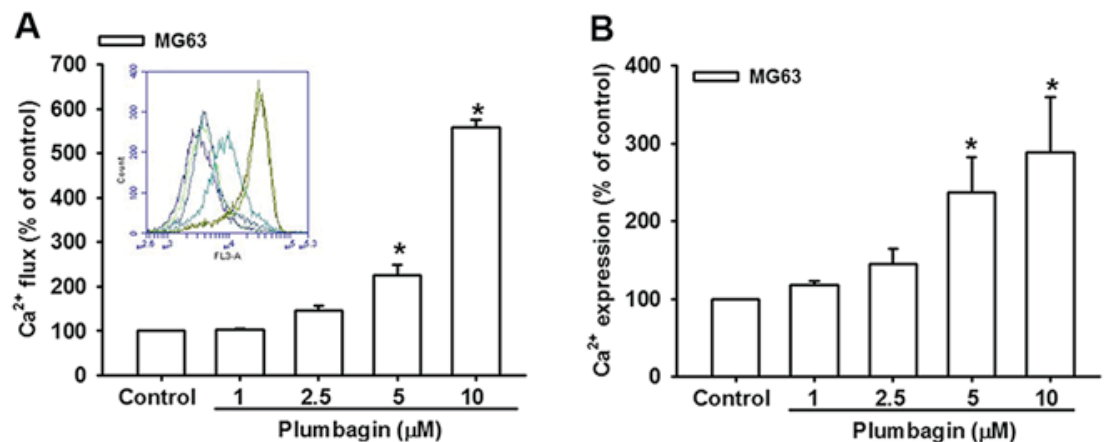

C
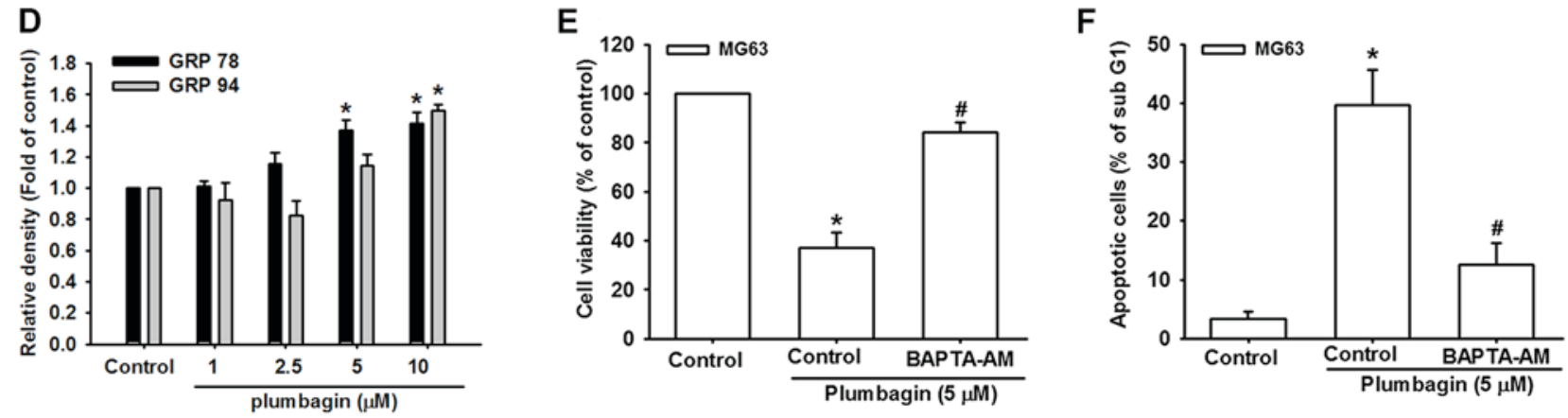

Figure 4. Plumbagin induces $\mathrm{Ca}^{2+}$ releasing and endoplasmic reticulum stress in human osteosarcoma cells. (A) MG63 cells were incubated with indicated concentrations of plumbagin for $24 \mathrm{~h}$. The cells were stained with Fluo 3/AM and $\mathrm{Ca}^{2+}$ release was assessed by flow cytometry. (B) MG63 cells were treated as described in (A), the cells were stained and $\mathrm{Ca}^{2+}$ expression was assessed using flow cytometry. (C) MG63 cells were treated as described in (A) and the protein expression levels of GRP78 and GRP94 were assessed by western blotting. (D) Quantitation of GRP78 and GRP94 protein levels in MG63 cells treated with 1, 2.5, 5 and $10 \mu \mathrm{M}$ plumbagin for $24 \mathrm{~h}$. MG63 cells were pretreated with BAPTA-AM (1 $\mu \mathrm{M})$ for $30 \mathrm{~min}$, and incubated with plumbagin for $24 \mathrm{~h}$. (E) Cell viability and (F) the percentage of apoptotic cells were assessed by propidium iodide staining and MTT assay. Results are expressed as the mean \pm standard error of the mean ${ }^{*} \mathrm{P}<0.05$ vs. control; ${ }^{*} \mathrm{P}<0.05$ vs. plumbagin-treated control; GRP, glucose-regulated protein.

\section{Results}

Plumbagin triggers cell apoptosis in human osteosarcoma cells. The authors first examined whether plumbagin could trigger apoptosis in osteosarcoma cells. Osteosarcoma cells (MG63, U2OS, HOS and hFOB1.19) were incubated with plumbagin for 24 or $48 \mathrm{~h}$, and the results indicated that plumbagin reduced cell viability in osteosarcoma cells but not normal bone cells (Fig. 1B). The $\mathrm{IC}_{50}$ values at $48 \mathrm{~h}$ of plumbagin were $0.59,0.78$ and $1.24 \mu \mathrm{M}$ for MG63, HOS and U2OS cells, respectively. The colony formation assay also consolidated the antitumor effect of plumbagin in osteosarcoma cells (Fig. 1C). Therefore, the authors investigated whether plumbagin induces apoptosis in osteosarcoma cells. The results indicated that plumbagin obviously increased DNA condensation (Fig. 1D). Degradation of chromatin was detected by a DNA ladder assay following plumbagin treatment (Fig. 2A). Cell apoptosis was also confirmed by cell cycle, Annexin V/PI double-labeling and TUNEL assay (Figs. 2B and C). To further verify the apoptosis induced by plumbagin, DNA fragmentation was conducted, as it is an important marker of apoptosis. It was observed that plumbagin induced cell apoptosis characterized by marked DNA fragmentation (Fig. 2D). These results support that plumbagin induces cell apoptosis in osteosarcoma cells.

ROS-triggered mitochondrial dysfunction is involved in plumbagin-induced apoptosis in osteosarcoma cells. ROS is known to induce mitochondrial dysfunction which promotes mitochondrial apoptotic pathway (18). Reactive oxygen species accumulation was detected after plumbagin treatment. The results demonstrated that $\mathrm{H}_{2} \mathrm{O}_{2}$ and ROS accumulation were increased following plumbagin treatment (Fig. 3A and B). Moreover, plumbagin-induced apoptosis was reversed by pretreating with ROS scavenger $\mathrm{N}$-acetyl cysteine (NAC), NADPH oxidase inhibitor diphenyleneiodonium chloride (DPI) and $\mathrm{H}_{2} \mathrm{O}_{2}$ scavenging enzyme (catalase) (Fig. 3C and D). JC-1 is a cationic dye that accumulates in energized mitochondria. In healthy cells, JC-1 accumulated as aggregates in the mitochondrial membranes, presenting a red to orange fluorescence. In unhealthy cells, a monomer that yields green fluorescence was shown. Treatment of cells with plumbagin induced marked changes in mitochondrial membrane proteins, as demonstrated by the disappearance of orange-red fluorescence, or by the increase of green fluorescence (Fig. 3E). The increase of the proapoptotic/antiapoptotic $\mathrm{Bcl}_{2}$ ratio and release of cytochrome c confirmed that the mitochondrial apoptosis pathway was induced by plumbagin treatment (Fig. 3F-K). In summary, these results suggested that plumbagin induces cell apoptosis through the ROS-triggered mitochondrial apoptotic pathway in osteosarcoma cells.

Endoplasmic reticulum (ER) stress is involved in plumbagin-induces apoptosis in human osteosarcoma cells. A previous study indicated that ER stress-related events are correlated with ROS generation such as unfolded protein response (19). Since $\mathrm{Ca}^{2+}$ release is highly associated with 

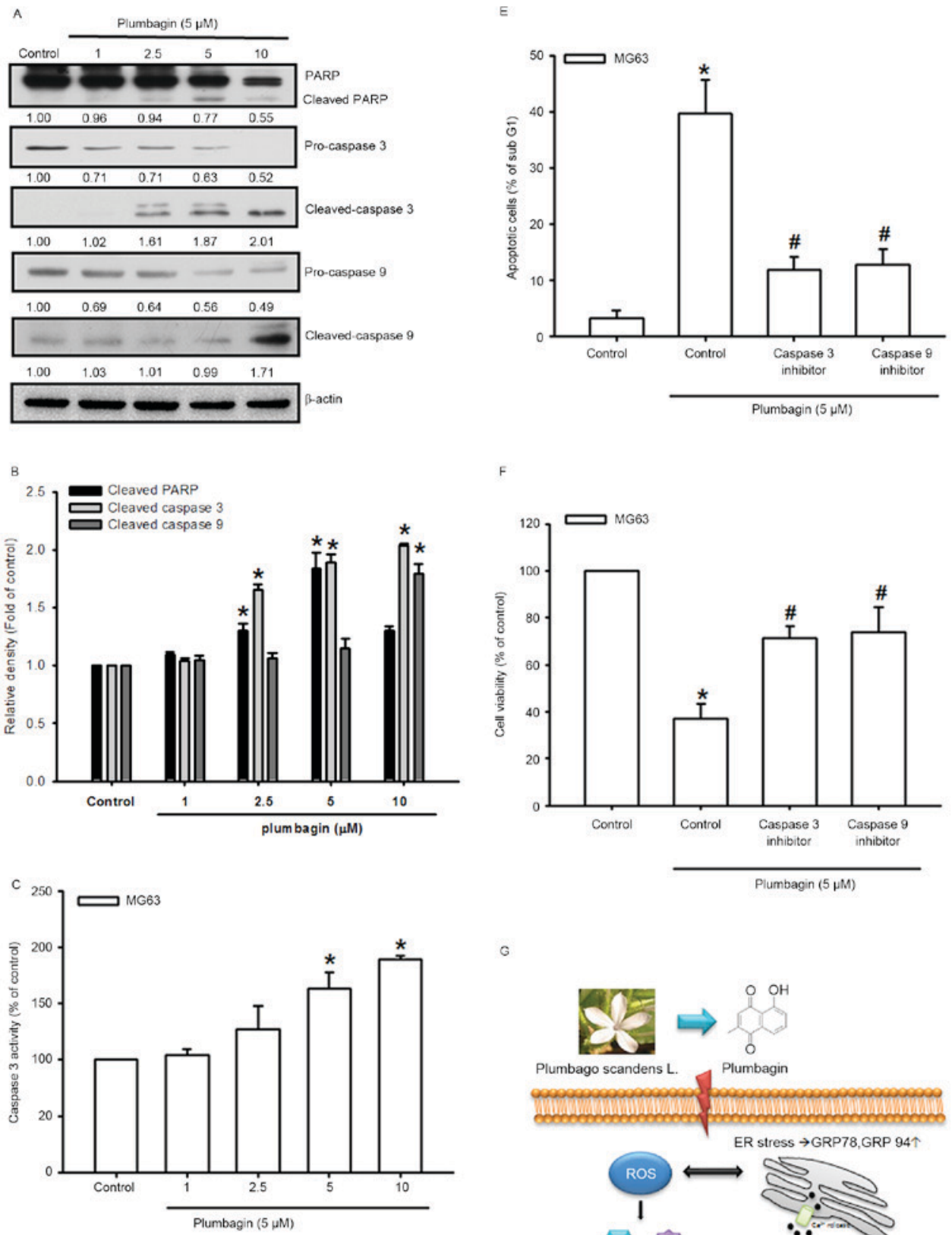

c
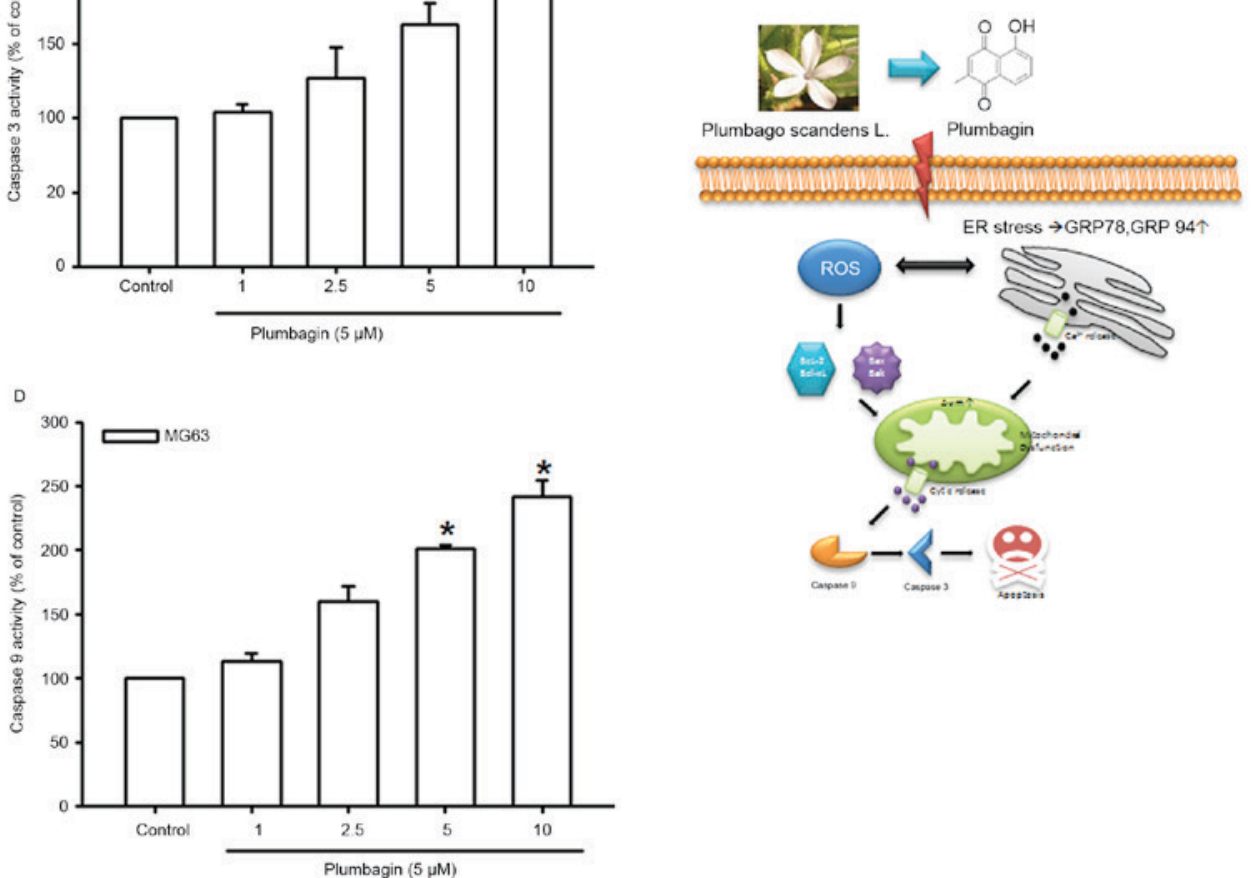

Figure 5. Plumbagin induces caspases activation in human osteosarcoma cells. (A) MG63 cells were treated with indicated concentrations of plumbagin for $24 \mathrm{~h}$. Total cell lysates were prepared, and the expression levels of PARP, caspase 3 and caspase 9 were assessed by western blot. $\beta$-actin was used as internal control. (B) Quantitation of cleaved PARP, cleaved caspase 3 and cleaved caspase 9 protein levels in MG63 cells treated with 1, 2.5, 5 and $10 \mu \mathrm{M}$ plumbagin for $24 \mathrm{~h}$. MG63 cells were treated as described in (A). The (C) caspase 3 and (D) caspase 9 activities were assessed using a caspase assay kit. MG63 cells were pretreated with caspase 3 and caspase 9 inhibitors and incubated with plumbagin for $24 \mathrm{~h}$. (E) The percentage of apoptotic cells and (F) cell viability were assessed by propidium iodide staining and MTT assay. (G) Schematic of plumbagin activity. Results are expressed as the mean \pm standard error of the mean ${ }^{*} \mathrm{P}<0.05$ vs. control; ${ }^{\#} \mathrm{P}<0.05$ vs. plumbagin-treated control. PARP, poly (ADP-ribose) polymerase; ROS, reactive oxidative species; ER, endoplasmic reticulum; GRP, glucose-regulated protein; Bcl, B cell lymphoma; Bak, Bcl-2 homologous antagonist/killer; Bax, Bcl-2-associated X; cyt c, cytochrome $c$. 
ER stress-related mitochondria dysfunction (20), the authors therefore examined $\mathrm{Ca}^{2+}$ accumulation following plumbagin incubation. The results demonstrated that plumbagin treatment increased intracellular calcium concentration (Fig. 4A and B). The results also indicated that GRP78 and GRP94, two major components involved in ER stress, were upregulated by plumbagin incubation (Fig. 4C and D). The authors confirmed this suggestion by pretreatment cell with cell-permeant chelator of calcium (BAPTA-AM), and the data showed that plumbagin-induced apoptosis was inhibited by pretreatment with BAPTA-AM (Fig. 4E and F). Conclusively, these data indicated that plumbagin induces apoptosis through the ER-stress pathway.

Plumbagin induced caspases activation in human osteosarcoma cells. Caspases are the critical components that regulate the apoptotic response. Finally, caspases activation was assessed following plumbagin incubation. Results indicated that caspase-3, caspase-9 and PARP (a downstream effector of caspase-3) were activated following plumbagin incubation (Fig. 5A and B). Plumbagin also increased caspase-3 and caspase-9 activity in osteosarcoma cells (Fig. 5C and D). Finally, pretreatment with caspase inhibitors abolished plumbagin induced cell apoptosis (Fig. 5E and F). These results suggested that caspase activation participates in plumbagin-induced cell apoptosis in osteosarcoma (Fig. 5G).

\section{Discussion}

Osteosarcoma is the most common bone malignancy that occurs in children. To date, chemotherapy and surgery are the only conventional treatment strategies. However, multidrug resistance limits the efficacy of chemotherapy in osteosarcoma and the 5-year survival rate is $20 \%$. Therefore, developing novel therapeutic strategies and improving prognosis is necessary. In the current study, the authors provide a new opportunity by using the natural product plumbagin in osteosarcoma therapy. Plumbagin induced cell apoptosis through activating ROS, ER stress, mitochondria dysfunction and caspase activation. In summary, the present study indicated that plumbagin may be a potential anticancer drug in osteosarcoma treatment.

Recently, the use of natural compounds in cancer therapy has become an alternative option. Plumbagin, the extract compound isolated from plants, has diverse biological functions such as antioxidant, anti-inflammatory, anti-bacterial and anticancer properties (21). Plumbagin demonstrates anticancer effects through various mechanisms. For example, plumbagin induces G2-M cell cycle arrest and autophagy of human breast cancer cells by targeting the Akt/mTOR pathway (6). In prostate cancer, plumbagin induces apoptosis through ROS generation and depletion of intracellular GSH levels. The authors reported that plumbagin-induced apoptosis was regulated by ROS generation. In addition, they indicated that plumbagin activated the mitochondrial apoptotic pathway, which, in turn, promoted the cleavage of caspase-9, caspase-3 and PARP. These investigations are in agreement with previous studies in lung cancer and pancreatic cancer $(22,23)$. Interestingly, the current finding indicated that plumbagin induced-apoptosis is correlated with ER-stress in osteosarcoma, and this effect has never been discussed before, to the best of the authors' knowledge.

Plumbagin demonstrates its anti-metastatic effects through migration and invasion inhibition in breast cancer (24). Moreover, plumbagin inhibits osteolytic bone metastasis by modulating the tumor-bone microenvironment in breast cancer $(25,26)$. However, little is understood about the anti-metastatic function of plumbagin in cancer progression. Recently, the bone microenvironment has been implicated in osteosarcoma progression. The interaction between osteosarcoma cells and resident cells such as osteoblasts, chondrocytes, mesenchymal stem/progenitor cells, hematopoietic cells and endothelial cells serve a central role in osteosarcoma progression (27). The role of plumbagin in osteosarcoma progression by modulating the bone microenvironment will be discussed in the future.

The conventional treatment strategy for osteosarcoma still leaves a poor prognosis. The present study reported the anticancer effect of plumbagin by inducing apoptosis in osteosarcoma cells. Plumbagin shows anticancer effects through multiple mechanisms in various tumors. These findings provide a novel insight into the action of plumbagin in osteosarcoma.

The antitumor effect of plumbagin was conducted by modulating multiple mechanisms in osteosarcoma. Plumbagin activated the apoptosis signaling pathway through eliciting ROS, ER stress, mitochondria dysfunction and causing caspase activation. In conclusion, these results indicated that plumbagin may serve as a potential antitumor drug by its multifunctional effects in osteosarcoma.

\section{Acknowledgments}

The present study was supported by grants from the National Science Council of Taiwan (grant nos. NSC-103-2314-B-341-004-MY3 and NSC-102-2314B-341-002-MY3) and Shin-Kong Wu Ho-Su Memorial Hospital (grant no. 104-SKH-FJU-14). The authors would like to thank the staff of the Eighth Core Lab, Department of Medical Research, National Taiwan University Hospital for technical support during the study.

\section{References}

1. Ek ET and Choong PF: The role of high-dose therapy and autologous stem cell transplantation for pediatric bone and soft tissue sarcomas. Expert Rev Anticancer Ther 6: 225-237, 2006.

2. Frankfurt OS and Krishan A: Apoptosis-based drug screening and detection of selective toxicity to cancer cells. Anticancer Drugs 14: 555-561, 2003.

3. Mossa JS, El-Feraly FS and Muhammad I: Antimycobacterial constituents from Juniperus procera, Ferula communis and Plumbago zeylanica and their in vitro synergistic activity with isonicotinic acid hydrazide. Phytother Res 18: 934-937, 2004.

4. Srinivas P, Gopinath G, Banerji A, Dinakar A and Srinivas G: Plumbagin induces reactive oxygen species, which mediate apoptosis in human cervical cancer cells. Mol Carcinog 40: 201-211, 2004.

5. Tilak JC, Adhikari S and Devasagayam TP: Antioxidant properties of Plumbago zeylanica, an Indian medicinal plant and its active ingredient, plumbagin. Redox Rep 9: 219-227, 2004.

6. Kuo PL, Hsu YL and Cho CY: Plumbagin induces G2-M arrest and autophagy by inhibiting the AKT/mammalian target of rapamycin pathway in breast cancer cells. Mol Cancer Ther 5: 3209-3221, 2006.

7. Aziz MH, Dreckschmidt NE and Verma AK: Plumbagin, a medicinal plant-derived naphthoquinone, is a novel inhibitor of the growth and invasion of hormone-refractory prostate cancer. Cancer Res 68: 9024-9032, 2008. 
8. Gomathinayagam R, Sowmyalakshmi S, Mardhatillah F, Kumar R, Akbarsha MA and Damodaran C: Anticancer mechanism of plumbagin, a natural compound, on non-small cell lung cancer cells. Anticancer Res 28: 785-792, 2008.

9. Ghobrial IM, Witzig TE and Adjei AA: Targeting apoptosis pathways in cancer therapy. CA Cancer J Clin 55: 178-194, 2005

10. Zapata JM, Pawlowski K, Haas E, Ware CF, Godzik A and Reed JC: A diverse family of proteins containing tumor necrosis factor receptor-associated factor domains. J Biol Chem 276: 24242-24252, 2001

11. Hockenbery D, Nuñez G, Milliman C, Schreiber RD and Korsmeyer SJ: Bcl-2 is an inner mitochondrial membrane protein that blocks programmed cell death. Nature 348: 334-336, 1990.

12. Mancini M, Nicholson DW, Roy S, Thornberry NA, Peterson EP, Casciola-Rosen LA and Rosen A: The caspase-3 precursor has a cytosolic and mitochondrial distribution: Implications for apoptotic signaling. J Cell Biol 140: 1485-1495, 1998.

13. Circu ML and Aw TY: Reactive oxygen species, cellular redox systems, and apoptosis. Free Radic Biol Med 48: 749-762, 2010.

14. Tang CH, Chiu YC, Huang CF, Chen YW and Chen PC: Arsenic induces cell apoptosis in cultured osteoblasts through endoplasmic reticulum stress. Toxicol Appl Pharmacol 241: 173-181, 2009.

15. Dijkers PF, Birkenkamp KU,Lam EW, Thomas NS, Lammers JW, Koenderman L and Coffer PJ: FKHR-L1 can act as a critical effector of cell death induced by cytokine withdrawal: Protein kinase B-enhanced cell survival through maintenance of mitochondrial integrity. J Cell Biol 156: 531-542, 2002.

16. Chen JT, Fong YC, Li TM, Liu JF, Hsu CW, Chang CS and Tang CH: DDTD, an isoflavone derivative, induces cell apoptosis through the reactive oxygen species/apoptosis signal-regulating kinase 1 pathway in human osteosarcoma cells. Eur J Pharmacol 597: 19-26, 2008.

17. Yang B and Reynolds CP: Tirapazamine cytotoxicity for neuroblastoma is p53 dependent. Clin Cancer Res 11: 2774-2780, 2005

18. Kowaltowski AJ, Castilho RF and Vercesi AE: Mitochondrial permeability transition and oxidative stress. FEBS Lett 495: $12-15,2001$.
19. Kitamura M and Hiramatsu N: The oxidative stress: Endoplasmic reticulum stress axis in cadmium toxicity. Biometals 23: 941-950, 2010.

20. Tabas I and Ron D: Integrating the mechanisms of apoptosis induced by endoplasmic reticulum stress. Nat Cell Biol 13: 184-190, 2011.

21. Padhye S, Dandawate P, Yusufi M, Ahmad A and Sarkar FH: Perspectives on medicinal properties of plumbagin and its analogs. Med Res Rev 32: 1131-1158, 2012.

22. Hsu YL, Cho CY, Kuo PL, Huang YT and Lin CC: Plumbagin (5-hydroxy-2-methyl-1,4-naphthoquinone) induces apoptosis and cell cycle arrest in A549 cells through p53 accumulation via c-Jun NH2-terminal kinase-mediated phosphorylation at serine 15 in vitro and in vivo. J Pharmacol Exp Ther 318: 484-494, 2006.

23. Chen CA, Chang HH, Kao CY, Tsai TH and Chen YJ: Plumbagin, isolated from Plumbago zeylanica, induces cell death through apoptosis in human pancreatic cancer cells. Pancreatology 9: 797-809, 2009

24. Yan W, Tu B, Liu YY, Wang TY, Qiao H, Zhai ZJ, Li HW and Tang TT: Suppressive effects of plumbagin on invasion and migration of breast cancer cells via the inhibition of STAT3 signaling and down-regulation of inflammatory cytokine expressions. Bone Res 1: 362-370, 2013.

25. Sung B, Oyajobi B and Aggarwal BB: Plumbagin inhibits osteoclastogenesis and reduces human breast cancer-induced osteolytic bone metastasis in mice through suppression of RANKL signaling. Mol Cancer Ther 11: 350-359, 2012.

26. Manu KA, Shanmugam MK, Rajendran P, Li F, Ramachandran L Hay HS, Kannaiyan R, Swamy SN, Vali S, Kapoor S, et al: Plumbagin inhibits invasion and migration of breast and gastric cancer cells by downregulating the expression of chemokine receptor CXCR4. Mol Cancer 10: 107, 2011.

27. Alfranca A, Martinez-Cruzado L, Tornin J, Abarrategi A, Amaral T, de Alava E, Menendez P, Garcia-Castro J and Rodriguez R: Bone microenvironment signals in osteosarcoma development. Cell Mol Life Sci 72: 3097-3113, 2015. 